|| ISSN(online): 2589-8698 || ISSN(print): 2589-868X || International Journal of Medical and Biomedical Studies

Available Online at www.ijmbs.info

PubMed (National Library of Medicine ID: 101738825)

Index Copernicus Value 2018: 75.71

Original Research Article

Volume 3, Issue 6; June: 2019; Page No. 279-283

\title{
CLINICAL ASSESSMENT OF SERUM TRIGLYCERIDE LEVELS IN PATIENTS SUFFERING FROM
} DIABETES IN AIIMS, PATNA

\section{Dr. Manish Kumar}

Senior Resident, Department of General Medicine, All India Institute of Medical Sciences, Patna, Bihar, India.

Article Info: Received 20 May 2019; Accepted 27 June. 2019

DOI: https://doi.org/10.32553/ijmbs.v3i6.408

Address for Correspondence: Dr. Manish Kumar

Conflict of interest: No conflict of interest.

\section{ABSTRACT}

India leads the world with largest number of diabetic patients and is often referred to as the diabetes capital. Diabetic dyslipidemia in India is one of the main causes for Coronary Artery Disease (CAD) mortality of the world. Dyslipidemia are disorders of lipoprotein metabolism, including lipoprotein overproduction or deficiency. It is a preventable risk factor which is mostly observed in diabetes patients and that may precipitate the cardiovascular disorders. Our aim of the study is to determine the impact of type 2 diabetes mellitus (T2DM) on lipid profile of diabetic patients.

The present study was planned in Department of General Medicine, All India Institute of Medical Sciences, Patna from july 2018 to December 2018. For the present study total 60 patients were selected. Out of 60 patients total 30 patients were enrolled in the group A as diabetic group and remaining 30 patients were enrolled in group B as normal patients. The biochemical parameters like Fating glucose level, Glycated haemoglobin (HbA1c), Total cholesterol, Triglycerides, High Density Lipid, and Low Density Lipid were estimated.

As diabetes is a disease of self-management, appropriate nutrition (low calories, low carbohydrates, and low fat with high fiber diet) regular physical activity and proper medication to achieve good glycaemic control have to be followed. Patients of diabetes with obesity- weight management are a key factor. The diabetic patients had elevated serum total cholesterol, elevated triglyceride. The slightly elevated low density lipoprotein (LDL-C) and reduced levels of high density lipoprotein (HDL-C) indicating that diabetic patients were more prone to cardiovascular diseases. Diabetes has now become a global endemic in both developing and developed countries. Hence it is the need of the hour for early detection and prevention of this non-communicable disease.

Keywords: Serum Triglyceride, Diabetes, LDL, HDL, etc.

\section{Introduction}

Diabetes is a chronic disease, which occurs when the pancreas does not produce enough insulin, or when the body cannot effectively use the insulin it produces. This leads to an increased concentration of glucose in the blood (hyperglycaemia). Type 1 diabetes (earlier known as insulin-dependent or childhood-onset diabetes) is characterized by a lack of insulin production. Type 2 diabetes (earlier known as non-insulin-dependent or adult-onset diabetes) is caused by the body's ineffective use of insulin. It often results from excess body weight and physical inactivity. Gestational diabetes is hyperglycaemia that is first recognized during pregnancy. It can lead to serious health risks for both the mother and child. Diabetes is a growing challenge in India with estimated $8.7 \%$ diabetic population in the age group of 20 and 70 years. The rising prevalence of diabetes and other noncommunicable diseases is driven by a combination of factors - rapid urbanization, sedentary lifestyles, unhealthy diets, tobacco use, and increasing life expectancy. [1]

Prevention and treatment involve maintaining a healthy diet, regular physical exercise, a normal body weight, and avoiding use of tobacco. Control of blood pressure and maintaining proper foot care are important for people with the disease. Type 1 diabetes must be managed with insulin injections. Type 2 diabetes may be treated with medications with or without insulin. Insulin and some oral medications can cause low blood sugar. Weight loss surgery in those with obesity is sometimes an effective measure in those with type 2 diabetes. Gestational diabetes usually resolves after the birth of the baby. Obesity and overweight are the most important risk factors responsible for diabetes. Much of the diabetes burden can be prevented or delayed 
by behavioural changes favouring a healthy diet and regular physical activity. [2]

The classic symptoms of untreated diabetes are unintended weight loss, polyuria (increased urination), polydipsia (increased thirst), and polyphagia (increased hunger). Symptoms may develop rapidly (weeks or months) in type 1 diabetes, while they usually develop much more slowly and may be subtle or absent in type 2 diabetes. Other symptoms of diabetes mellitus include weight loss and tiredness. Several other signs and symptoms can mark the onset of diabetes although they are not specific to the disease. In addition to the known ones above, they include blurred vision, headache, fatigue, slow healing of cuts, and itchy skin. Prolonged high blood glucose can cause glucose absorption in the lens of the eye, which leads to changes in its shape, resulting in vision changes. Long-term vision loss can also be caused by diabetic retinopathy. A number of skin rashes that can occur in diabetes are collectively known as diabetic dermadromes.[3]

In the human body, high levels of triglycerides in the bloodstream have been linked to atherosclerosis, heart disease and stroke. However, the relative negative impact of raised levels of triglycerides compared to that of LDL:HDL ratios is as yet unknown. The risk can be partly accounted for by a strong inverse relationship between triglyceride level and HDL-cholesterol level. But the risk is also due to high triglyceride levels increasing the quantity of small, dense LDL particles.[4]

Current recommendations for cholesterol testing come from the Adult Treatment Panel (ATP) III guidelines, and are based on many large clinical studies, such as the Framingham Heart Study. For healthy adults with no cardiovascular risk factors, the ATP III guidelines recommend screening once every five years.[5] A lipid profile may also be ordered at regular intervals to evaluate the success of lipidlowering drugs such as statins. In the pediatric and adolescent population, lipid testing is not routinely performed. However, the American Academy of Pediatrics and NHLBI now recommend that children aged $9-11$ be screened once for severe cholesterol abnormalities.[6] This screening can be valuable to detect genetic diseases such as familial hypercholesterolemia that can be lethal if not treated early. Traditionally, most laboratories have required patients to fast for 9-12 hours before screening. However, recent studies have questioned the utility of fasting before lipid panels, and some diagnostic labs now routinely accept non-fasting samples.[7]

This test is used to identify dyslipedemia (various disturbances of cholesterol and triglyceride levels), many forms of which are recognized risk factors for cardiovascular disease and rarely pancreatitis. A total cholesterol reading can be used to assess an individual's risk for heart disease, however, it should not be relied upon as the only indicator. The individual components that make up total cholesterol reading-LDL, HDL, and VLDL-are also important in measuring risk. For instance, someone's total cholesterol may be high, but this may be due to very high HDL ("good cholesterol") cholesterol levels, which can actually help prevent heart disease (the test is mainly concerned with high LDL, or "bad cholesterol" levels). So, while a high total cholesterol level may help give an indication that there is a problem with cholesterol levels, the components that make up total cholesterol should also be measured.

Weight loss and dietary modification are effective first-line lifestyle modification treatments for hypertriglyceridemia. For people with mildly or moderately high levels of triglycerides lifestyle changes including weight loss, moderate exercise and dietary modification are recommended. This may include restriction of carbohydrates (specifically fructose) and fat in the diet and the consumption of omega-3 fatty acids from algae, nuts, and seeds. Medications are recommended in those with high levels of triglycerides that are not corrected with the aforementioned lifestyle modifications, with fibrates being recommended first. Epanova (omega-3carboxylic acids) is another prescription drug used to treat very high levels of blood triglycerides.[8]

The decision to treat hypertriglyceridemia with medication depends on the levels and on the presence of other risk factors for cardiovascular disease. Very high levels that would increase the risk of pancreatitis is treated with a drug from the fibrate class. Niacin and omega- 3 fatty acids as well as drugs from the statin class may be used in conjunction, with statins being the main medication for moderate hypertriglyceridemia when reduction of cardiovascular risk is required.[9]

Glycated haemoglobin (HbA1c) is a routinely used marker for long-term glycaemic control. Apart from functioning as an indicator for the mean blood glucose level, HbA1c also predicts the risk for the development of diabetic complications in diabetes 
patients. [10] Many studies have proposed HbA1c to be used as a biomarker of both glycaemic control and dyslipidemia in type 2 diabetes mellitus. [11-13]

India leads the world with largest number of diabetic patients and is often referred to as the diabetes capital. Diabetic dyslipidemia in India is one of the main causes for Coronary Artery Disease (CAD) mortality of the world. Dyslipidemia are disorders of lipoprotein metabolism, including lipoprotein overproduction or deficiency. It is a preventable risk factor which is mostly observed in diabetes patients and that may precipitate the cardiovascular disorders. Our aim of the study is to determine the impact of type 2 diabetes mellitus (T2DM) on lipid profile of diabetic patients.

\section{Methodology:}

The present study was planned in Department of General Medicine, All India Institute of Medical Sciences, Patna from July 2018 to December 2018.For the present study total 60 patients were selected. Out of 60 patients total 30 patients were enrolled in the group $A$ as diabetic group and remaining 30 patients were enrolled in group $B$ as normal patients. The biochemical parameters like Fasting glucose level, Glycated haemoglobin (HbA1c), Total cholesterol, Triglycerides, High Density Lipid, and Low Density Lipid were estimated.

All the patients were informed consents. The aim and the objective of the present study were conveyed to them. Approval of the institutional ethical committee was taken prior to conduct of this study.

Following was the inclusion and exclusion criteria for the present study.

Inclusion criteria: Type 2 diabetes mellitus patients in the age range of $20-85$ years.

Exclusion criteria: T2DM patients with concomitant diseases or conditions affecting lipid levels like chronic liver disease and hypothyroidism. Patients on drugs like oral contraceptive pills, steroids and diuretics. Smokers, alcoholics, patients with history of liver and renal impairment were excluded from the study.

\section{Results \& Discussion:}

Dyslipidemia was defined by presence of one or more than one abnormal serum lipid concentration [10]. In patients with diabetes, many studies have clearly established that complications are mainly due to chronic hyperglycemia that exerts its injurious to health effects through several mechanisms: dyslipidemia, platelet activation, and altered endothelial metabolism [14-15]. Both lipid profile and diabetes have been shown to be the important predictors for metabolic disturbances including dyslipidemia, hypertension and cardiovascular diseases [16].

For the interpretation of serum lipid reference values, the guidelines of National Cholesterol Education Programme (NCEP) Adult Treatment Panel III (ATP III) were followed. According to NCEP-ATPIII guidelines, hypercholesterolemia is defined as TC $>200 \mathrm{mg} / \mathrm{dl}$, high LDL-C when value > $100 \mathrm{mg} / \mathrm{dl}$, hypertriglyceridemia as TAG > $150 \mathrm{mg} / \mathrm{dl}$ and low $\mathrm{HDL}-\mathrm{C}$ when value is $<40 \mathrm{mg} / \mathrm{dl}$. Dyslipidemia was defined by presence of one or more than one abnormal serum lipid concentration. [16]

Lipids play a vital role in the pathogenesis of diabetes mellitus. Dyslipidemia as a metabolic abnormality is frequently associated with diabetes mellitus. Abnormalities in lipid metabolism have been reported in patients with diabetes mellitus accompanied by the risk of cardiovascular arteriosclerosis [14]. In the present study, significantly higher mean serum levels of total cholesterol, triglycerides were noted in patients with diabetes, which are well known risk factors for cardiovascular diseases among patients, when compared to the normal values.

Senthilkumar et al [17], conducted a perspective study on 162 type 2 diabetes mellitus patients in Tamil Nadu. They found no significant correlation of HbA1c with TC, LDL, HDL and TG.

Jayesh et al [18] conducted a prospective study on western Indian population that comprised of 430 type 2 diabetes mellitus patients and 501 non diabetic control subjects. They found significant correlation of $\mathrm{HbA} 1 \mathrm{c}$ with TC and LDL.

Table 1: Comparison of General Parameter

\begin{tabular}{|l|l|l|}
\hline Group & Group A & Group B \\
\hline $\begin{array}{l}\text { Type of } \\
\text { Patients }\end{array}$ & $\begin{array}{l}\text { Diabetic } \\
\text { patients }\end{array}$ & $\begin{array}{l}\text { Controlled study } \\
\text { patients }\end{array}$ \\
\hline No. of Patients & 30 & 30 \\
\hline Age Group & $38-52$ years & $34-55$ year \\
\hline Males & 23 & 20 \\
\hline Females & 7 & 10 \\
\hline
\end{tabular}


Table 2: Comparison of Bio Chemical Parameter

\begin{tabular}{|l|l|l|}
\hline Group & Group A & Group B \\
\hline & $\begin{array}{l}\text { Diabetic } \\
\text { patients }\end{array}$ & $\begin{array}{l}\text { Controlled } \\
\text { study } \\
\text { patients }\end{array}$ \\
\hline Type of Patients & 30 & 30 \\
\hline Bio Chemical Parameter & Observation \\
\hline Triglycerides (mg\%) & $196.5 \pm 25.9$ & $179.2 \pm 23.3$ \\
\hline Fasting glucose level (mg\%) & $161.2 \pm 8.6$ & $95.7 \pm 6.4$ \\
\hline $\begin{array}{l}\text { Glycated haemoglobin } \\
\text { (HbA1c) (\%) }\end{array}$ & $8.6 \pm 1.2$ & $6.7 \pm 0.9$ \\
\hline Total cholesterol (mg\%) & $183.2 \pm 12.6$ & $168.5 \pm 14.3$ \\
\hline High Density Lipid (mg\%) & $41.6 \pm 5.6$ & $50.3 \pm 7.1$ \\
\hline Low Density Lipid (mg\%) & $119.2 \pm 16.3$ & $93.5 \pm 19.5$ \\
\hline
\end{tabular}

Zhe Yan et al [19] conducted a study on 128 type 2 diabetes mellitus patients in Sichuan, China. They found significant correlation of $\mathrm{HbA1c}$ with LDL.

Eglal et al [20] a study on 50 type 2 diabetes mellitus patients in Khartoum Sudan, they found significant correlation of $\mathrm{HbA} 1 \mathrm{c}$ with TG.

Diabetic patients have many complications which include elevated levels of LDL-C and triacylglycerols, low levels of HDL-C and a preponderance of abnormalities in the composition of the smaller, dense particles [21]. Similar findings found in study done by Idogun, et al. [22] and Albrki, et al. [23] and observed that lipoprotein profiles of the diabetics were found higher than normal reference values.

The control rates for dyslipidemia when all the four lipid parameters are considered together, showed a grim picture with approximately $6 \%$ of the total population achieving goals. There was no significant difference between control rates of lipid levels across the four zones in India.

Moreover, the study reveals that patients with diabetes and overt CVD are not achieving guideline recommended target LDL-C levels. Considering the rising prevalence and changing epidemiology of both diabetes and CVD and the higher likelihood of their coexistence in India, this data provide important insights on control of dyslipidemia in this vulnerable population. Therefore, this calls for immediate attention by the medical community to resort to a more aggressive approach to manage dyslipidemia, especially in those with diabetes and overt CVD.

Based on the study results, it is clear that aggressive dyslipidemia management is the need of the hour in patients with diabetes. We hope our study will pave the way for future research in this area and also help the medical fraternity in consciously taking measures to address these burning issues.

Hyperlipidemia and altered lipid metabolism is commonly seen in diabetes. The relationship between elevation of serum lipids and vascular complication of diabetes has long been of interest because both tend to occur with greater frequency in diabetes mellitus than in general population. The increased risk of vascular disease in diabetic patients may be in part due to the associated hyperlipidemia [24].

\section{Conclusion:}

As diabetes is a disease of self-management, appropriate nutrition (low calories, low carbohydrates, and low fat with high fiber diet) regular physical activity and proper medication to achieve good glycaemic control have to be followed. Patients of diabetes with obesity- weight management are a key factor. The diabetic patients had elevated serum total cholesterol, elevated triglyceride. The slightly elevated low density lipoprotein (LDL-C) and reduced levels of high density lipoprotein (HDL-C) indicating that diabetic patients were more prone to cardiovascular diseases. Diabetes has now become a global endemic in both developing and developed countries. Hence it is the need of the hour for early detection and prevention of this non-communicable disease.

\section{References:}

1. http://www.searo.who.int/india/topics/diabetes_mel litus/en/

2. Cash, Jill (2014). Family Practice Guidelines (3rd ed.). Springer. p. 396. ISBN 978-0-8261-6875-7. Archived from the original on 31 October 2015.

3. Rockefeller, J.D. (2015). Diabetes: Symptoms, Causes, Treatment and Prevention. ISBN 978-1-5146-0305-5.

4. Ivanova EA, Myasoedova VA, Melnichenko AA, Grechko AV, Orekhov AN (2017). "Small Dense LowDensity Lipoprotein as Biomarker for Atherosclerotic Diseases". Oxidative Medicine and Cellular Longevity. 2017: 1273042. doi:10.1155/2017/1273042. PMC 5441126. PMID 28572872

5. National Cholesterol Education Program (Ncep) Expert Panel On Detection, E. (2002). "Third Report of the National Cholesterol Education Program (NCEP) Expert Panel on Detection, Evaluation, and Treatment of High Blood Cholesterol in Adults (Adult Treatment Panel III) final report". Circulation. 106 (25): 31433421. PMID 12485966. 
6. "Pediatric Cardiovascular Risk Reduction Guidelines NHLBI, NIH". Archived from the original on 2012-1116.

7. Sidhu, D.; Naugler, C. (2012). "Fasting Time and Lipid Levels in a Community-Based Population: A Crosssectional Study / Fasting Time and Lipid Levels". Archives of Internal Medicine. 172(22): 1-4. doi:10.1001/archinternmed.2012.3708. PMID 23147400.

8. Blair HA, Dhillon S. Omega-3 carboxylic acids (Epanova): a review of its use in patients with severe hypertriglyceridemia. Am J Cardiovasc Drugs. 2014 Oct;14(5):393-400. PMID 25234378

9. Berglund L, Brunzell JD, Goldberg AC, et al. (September 2012). "Evaluation and treatment of hypertriglyceridemia: an endocrine society clinical practice guideline". J. Clin. Endocrinol. Metab. 97 (9): 2969-89. doi:10.1210/jc.2011-3213. PMC 3431581. PMID 22962670

10. Selvin E. Meta-Analysis: Glycosylated hemoglobin and cardiovascular disease in diabetes mellitus. Annals of Internal Medicine. 2004;141:421.

11. Lodha $R$, Lal $R$, Biyani $S$. HbA1c as screening biomarker of dyslipidemia in type 2 diabetes mellitus patients. Scholar journal of applied medicsl sciences. 2016;4:1600-1602.

12. Alan R, Verma P. Glycated hemoglobin as a dual biomarker in type 2 diabetes mellitus predicting glycemic control and dyslipidemia risk. International journal of life science scientific research. 2015;1:6265.

13. Parveen A, Chimkode SM, Kumaran SD. Correlation of $\mathrm{HbA1c}$ levels with serum lipid profile in patients with type 2 diabetes mellitus. Research journal of pharmaceutical, biological and chemical sciences. 2015;6:703-706.

14. Brownlee M. Biochemistry and molecular cell biology of diabetic complications. Nature, 2001, 414(6865): 813820 .
15. Taskinen MR. Diabetic dyslipidaemia: from basic research to clinical practice. Diabetologia, 2003; 46(6): 733-749.

16. Goldberg IJ. Diabetic dyslipidemia: causes and consequences. J Clin Endocr Metab., 2001; 8(3): 965971.

17. Senthilkumar N, Anadasayanam A, Senthilvelu S, Rashid M. Correlation observation between HbA1C and Lipid profile in Type II Diabetes Mellitus OutPatients. International Journal of Pharma Research and Review, 2016;5:9-20.

18. Sheth J, Shah A, Sheth F, Trivedi S, Nabar N, Shah N et al. The association of dyslipidemia and obesity with glycated hemoglobin. Clinical Diabetes and Endocrinology. 2015;1(1).

19. Yan Z, Liu Y, Huang H. Association of glycosylated hemoglobin level with lipid ratio and individual lipids in type 2 diabetic patients. Asian Pacific Journal of Tropical Medicine. 2012;5:469-471.

20. Abd Elkarim A. Abdrabo et al. Role of glycemic control on lipids profile in diabetic sudanese patients. Journal of Science. 2016;6:208-212.

21. Sacks FM, Hermans MP, Fioretto $P$, Valensi $P$, Davis $T$, Horton E, Wanner C, Al-Rubeaan K, Aronson R, Barzon I, Bishop L. Association Between Plasma Triglycerides and High-Density Lipoprotein Cholesterol and Microvascular Kidney Disease and Retinopathy in Type 2 Diabetes Mellitus. Circulation, 2014 Mar 4; 129(9): 999- 1008.

22. Idogun ES, Unuigbe El, Ogunro PS, Akinola OT, Famodu AA. Assessment of the serum lipids in Nigerians with type 2 diabetes mellitus complications. Pak. J. Med. Sci. (Part 1), 2007; 23(5): 708-12.

23. Albrki WM, Elzouki AN Y, ELMansoury ZM, Tashani OA. Lipid profiles in Libian type 2 diabetes. J. Sci. Appls., 2007; 1(1): 18-23.

24. Suri, R.K., Guptha, M.M. and Chakkravarthi, A.K. Hyper-lipidaemias and vascular populations of diabetes mellitus. J. Ass. Phys. India., 27: 505 (1979). 